\section{Patientensicherheit kann optimiert werden}

\author{
Interview mit Dr. Beat Meister, Stiftungsratspräsident
} der Stiftung für Patientensicherheit in der Anästhesie

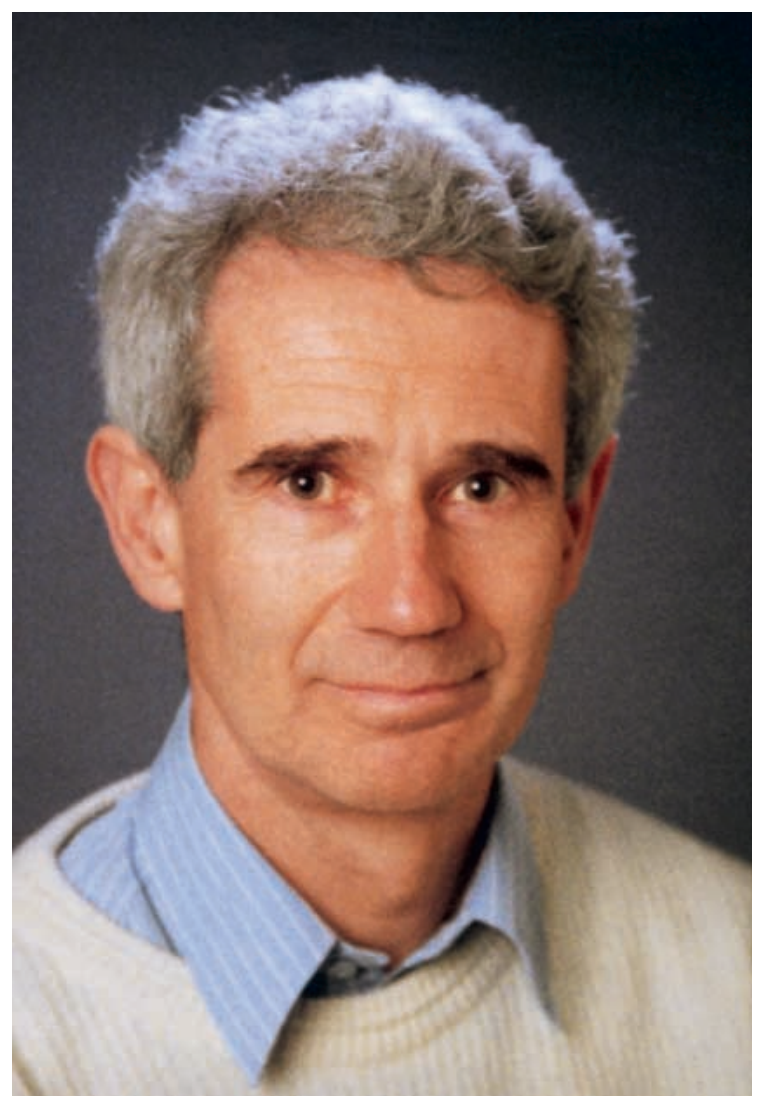

"Ärzte wollen aus Fehlern lernen, aber ohne dass sie gleich vor den Strafrichter müssen» (Tages-Anzeiger). «Eine von der Schweizerischen Gesellschaft für Anästhesiologie und Reanimation (SGAR) initiierte 'Stiftung für Patientensicherheit in der Anästhesies hat zum Ziel, für alle Patienten eine optimale Sicherheit während der Narkose zu erreichen» (NZZ): Das nationale Medienecho auf die Initiative der Schweizer Anästhesisten war gross, passte millimetergenau in die laufende Diskussion zum Thema Ärztefehler und setzte so einen Gegenpunkt zur Negativberichterstattung. Denn es ist das erste Mal, dass Mediziner mit nichtmedizinischen Partnern zusammenarbeiten, um nicht nur eine heikle Thematik zu diskutieren, sondern auch umfassend anzugehen. In der Trägerschaft der Stiftung sind neben der SGAR auch Patientenorganisationen, Versicherungen sowie pharmazeutische und medizinaltechnologische Firmen vertreten. Wir haben uns mit dem Stiftungsratspräsidenten, Dr. Beat Meister, Bern, über die Stiftung und deren Ziele und Auswirkungen unterhalten.

Schweizerische Ärztezeitung: Medizinische Behandlungsfehler dominieren die Schlagzeilen in den Medien. Es scheint so, dass Sie mit Ihrer Stiftung gerade rechtzeitig an die Öffentlichkeit gelangen ...

Dr. Beat Meister: ... glauben Sie mir: Ich hätte mir für die Präsentation unserer Stiftung ein weniger tragisches Umfeld gewünscht. Aber es ist schon so. Wir haben eine Antwort auf eine aktuelle Frage. Wir können das, was an der FMH-Tagung am 25. Februar in Bern diskutiert wurde, konkretisieren. Bekanntlich will die FMH «Kunstfehler» des medizinischen Personals künftig in einer Datenbank festhalten. Gemäss FMH-Präsident Brunner sollen begangene Fehler akzeptiert, soll mit ihnen umgegangen werden können.

Was wollen Sie mit der Stiftung für Patientensicherheit in der Anästhesie erreichen?

Ziel und Zweck der Stiftung sind unmissverständlich: Projekte zur Verbesserung der Patientensicherheit zu unterstützen. Es sollen möglichst viele potentielle und effektive Zwischenfälle erfasst und analysiert werden. Diese Erkenntnisse wiederum werden in Fachkreisen kommuniziert und damit thematisiert. Es sollen aber auch Aktivitäten zur Verhinderung von Zwischenfällen unterstützt werden. Kurz: Durch die Stiftung wird ein grosser Beitrag zur Verhinderung von potentiellen Fehlern geleistet.
Dr. Beat Meister, 52 Jahre, ist Präsident der Stiftung für Patientensicherheit in der Anästhesie sowie ehemaliger Präsident der Schweizerischen Gesellschaft für Anästhesiologie und Reanimation. Er ist als Spezialarzt für Anästhesie und Intensivmedizin FMH in der Klinik Beau-Site in Bern tätig.

Bekanntlich lassen sich Fehler oft nicht vermeiden, wohl aber deren Wiederholung. Wie gehen Sie nun konkret vor?

Einleitend möchte ich festhalten: Grösster Risikofaktor ist der Zeitdruck, unter dem wir Anästhesieärzte mitunter arbeiten müssen: Durch die sich verschlechternden Rahmenbedingungen wird das Risiko eindeutig erhöht. Die Anästhesie ist ein besonders heikler Bereich, weil beispielsweise vor einer Operation schnell wirkende und starke Medikamente verabreicht und die Patienten anschliessend künstlich beatmet werden. Die kleinsten Fehler können gravierende Folgen haben.

Zur Verhinderung von Zwischenfällen und zur Aufarbeitung des Geschehenen müssen entsprechende Strukturen geschaffen werden. Aus dieser Erkenntnis heraus hat die SGAR bereits 1996 zwei grosse Projekte gestartet. Das erste ist das sogenannte "Critical Incidents Report System" (CIRS). Das CIRS ist eine Pionierleistung, die von Dr. Sven Staender, (heutiger Chefarzt für Anästhesiologie und Intensivmedizin in Männedorf) bereits vor 10 Jahren an der Universität Basel mit Unterstützung von Prof. Daniel Scheidegger, begonnen wurde. In Anlehnung an ähnliche Meldesysteme aus der Aviatik und an ein 
System zur Meldung von Anästhesiezwischenfällen in Australien, wurde ein anonymes, elektronisches Meldesystem für die Schweizer Anästhesie (CIRS-CH) entwickelt. Das System erlaubt jedem Anästhesiearzt und jeder Anästhesiepflegeperson via Internet jederzeit kritische Ereignisse zu melden, anzuschauen und zu kommentieren. Das zweite Projekt analysiert die abgeschlossenen Haftpflichtfälle und wird primär von Prof. Hansjürg Schär, Männedorf, betreut und orientiert sich weitgehend am "anesthesia closed claims project» der Amerikaner. Von beiden Projekten werden die Fälle von einem Expertengremium überarbeitet und die Erkenntnisse daraus in geeigneter Weise, vollständig anonymisiert, publiziert. Falls notwendig, werden entsprechende Massnahmen ergriffen, Firmen über Produkteprobleme informiert oder neue Sicherheitsstandards geschaffen.

Ein Novum ist, dass in der "Stiftung für Patientensicherheit in der Anästhesie» medizinische und nichtmedizinische Partner zusammenarbeiten. Was erhoffen Sie sich davon?

Wir haben ein gemeinsames Ziel: so wenig Zwischenfälle wie möglich. Es freut mich auch, dass unter Umständen ein weiterer wichtiger Partner zu uns stossen wird: Das Konkordat der Schweizerischen Krankenkassen hat sein Interesse an einer Trägerschaft angemeldet. Gemeinsam verfügen wir über ein immenses Potential an Know-how, Infrastruktur und Durchsetzungskraft. Mich freut vor allem aber, dass die Schweizer Patientenorganisation mit von der Partie ist. Es geht nämlich primär um den Patienten und daher ist es für mich ausserordentlich wichtig, dass die Direktbetroffenen nun endlich mit dabei sind.

Den Medien ist zu entnehmen, dass Sie auch auf politischer Ebene aktiv werden wollen.

Ein zentrales Anliegen ist die Entkriminalisierung von Zwischenfällen. Ärztefehler sollen nicht mehr als Offizial-, sondern nur als Antragsdelikt taxiert werden. Das heisst, dass ein Arzt nur auf Antrag des Betroffenen oder dessen Angehörigen strafrechtlich verfolgt würde.

Ein zweites wichtiges Anliegen ist es, dem Qualitätsabbau im gesundheitspolitischen Umfeld Einhalt zu gebieten. Von den Medizinalpersonen, dem Pflegepersonal wie von den Ärzten wird immer mehr gefordert, ohne dass entsprechende Ressourcen - und dabei meine ich hauptsächlich Zeit - zur Verfügung gestellt werden. Chefärzte, die noch mehr Patienten in noch weniger Zeit behandeln, sind zur Zeit die eigentlichen Helden im Gesundheitswesen, wofür sie von Spital- und Gesundheitsdirektoren gelobt werden. Medizin ist aber keine Akkordarbeit! Durch den permanenten Zeitdruck und durch ungenügende personelle Dotierungen wird das Risiko für Zwischenfälle eindeutig erhöht.
Die Stiftung wird von den folgenden Institutionen und Firmen getragen

Schweizerische Gesellschaft für Anästhesiologie (SGAR)

- Schweizerische Patientenorganisation (SPO)

- Schweizerischer Versicherungsverband (SVV)

- Astra Zeneca

- Abbott

- AVL Medical Instruments

- Carbamed

- GlaxoSmithKline

Organon Teknika

Kommen wir nochmals auf Ihr "Critical Incidents"Modell zu sprechen. Welche Erfahrungen haben Sie bei der Auswertung von Zwischenfällen gemacht?

Als Ursache für das Fehlverhalten nannten die Betroffenen in 64 Prozent der Fälle Übermüdung, Unachtsamkeit, Hektik, unterlassene Kontrollen und falsche Beurteilung der Situation. Bei 20 Prozent war mangelnde Kommunikation der Grund. Technische Probleme sind also nur in einem kleinen Teil der Fälle für einen Behandlungsfehler verantwortlich.

Ein Beispiel: Eine notfallmässige Anästhesie bei einem Patienten in einem septischen Schock musste wegen Personalmangel durch einen Assistenten im ersten Jahr seiner Ausbildung vorgenommen werden. Die Tragweite eines lange anhaltenden Blutdruckabfalles wurde dabei nicht realisiert. Der Patient ist deshalb zerebral geschädigt und teilinvalid. Konsequenz für die Sicherheit: Die Stellenpläne und Organigramme in den Kliniken müssen so angelegt sein, dass für alle Anästhesien ausreichend qualifiziertes Personal verfügbar ist.

Apropos Politik: Wie beurteilen Sie die Rolle des Bundesamtes für Sozialversicherungen (BSV)?

Anlässlich unserer Medienorientierung zur Stiftungsgründung begrüsste Manfred Langenegger, zuständig für Qualitätssicherung beim BSV, das Engagement der Anästhesisten. Er attestierte ihnen eine Vorreiterrolle im Bereich Fehlermanagement. Dies darf aber nicht darüber hinwegtäuschen, dass die hohe Medienpräsenz der "Kunstfehler» durch die Aussage des BSV ausgelöst wurde, in der Schweiz würden jährlich etwa 3000 Spitalpatienten wegen Behandlungsfehlern sterben. Ich habe den Eindruck, dass es das Ziel des BSV ist, auf staatlicher Ebene einen Qualitätskontrollapparat zu institutionalisieren. Für mich unverständlich ist, weshalb die 1998 eingereichte und vom Bundesrat gutgeheissene Motion Günter zur Aufarbeitung aller schweren medizinischen Zwischenfälle ausgerechnet 
im Departement Dreifuss einfach schubladisiert wurde. Wir wehren uns keineswegs gegen ein vernünftiges Aufarbeiten unserer Fehler. Im BSV ist diese Kommission, die die Ärzte überwachen soll, für mich jedoch am falschen Ort. Das BSV tut alles für eine Verstaatlichung der Medizin und das kann nur kontraproduktiv sein. Für mich gehört die Kommission zur Analyse von medizinischen Zwischenfällen ins BAG und sicher nicht ins BSV, denn es geht primär um die Medizin und nicht um die Finanzen.

Wie haben die Medien, die ja gerne über Behandlungsfehler in der Medizin berichten, die Idee Ihrer Stiftung aufgenommen?

Wir hatten in allen Landesteilen bei den Print- und elektronischen Medien ein sehr gutes Echo: So haben u. a. Tages-Anzeiger, NZZ, Basler Zeitung, Weltwoche, Tribune Médicale, Le Temps, Le Matin, Corriere del Ticino, Schweizer Fernsehen (Tagesschau), Schweizer Radio DRS sowie verschiedene Lokalradios und Fernsehstationen über die Lancierung unserer Stiftung be- richtet. Es war von einem «Frühwarnsystem für Ärzte» die Rede, welches die Anästhesisten auf dem Weg "von der Schuldfrage zur Fehlerkultur» unterstützt. Auch kamen konkrete Zahlen zur Sprache: Das Risiko, bei der Fahrt ins Spital ums Leben zu kommen (1:10 000) ist ungleich grösser als dasjenige, bei einem Anästhesiezwischenfall sein Leben zu verlieren (1:40 000 bis 1:200 000). Wir konnten unsere Botschaften optimal positionieren und uns gegenüber den Medienschaffenden als Informationspartner profilieren. Die Berichterstattung ist für uns eine Herausforderung, laufend über unsere Erfahrungen zu berichten.

\section{Haben Sie einen besonderen Wunsch?}

Ich wünsche mir, dass die Grundzüge der Stiftung und ihrer Tätigkeit auch von anderen Ärztegruppen aufgenommen werden und ihre Resonanz auch innerhalb der FMH finden.

Herr Dr. Meister, vielen Dank für dieses Gespräch. 